\title{
Bacterial infections in cirrhosis: a narrative review and key points for clinical practice
}

\author{
Mario Mitra, Andrea Mancuso, Flavia Politi, Alberto Maringhini \\ Medicina Interna 1, ARNAS Civico-Di Cristina-Benfratelli, Palermo, Italy
}

\begin{abstract}
Bacterial infections are frequent complications of liver cirrhosis, accounting for severe clinical courses, and increased mortality. The reduction of the negative clinical impact of infections may be achieved by a combination of prophylactic measures to reduce the occurrence, early identification, and management. Spontaneous bacterial peritonitis (SBP), urinary tract infections, pneumonia, cellulitis, and spontaneous bacteremia are frequent in cirrhosis. The choice of initial empirical antimicrobial therapy should be based on both site, severity, and origin of infection (community-acquired, nosocomial, or healthcare-associated) and on antibiotic resistance patterns. $3^{\text {rd }}$ generation cephalosporins are generally indicated as empirical therapy in most community-acquired cases. However, for nosocomial and healthcare-associated infections, due to a high rate of multidrug-resistant (MDR) pathogens, a broader spectrum treatment is appropriate. In order to prevent antibiotic resistance emergence, microbiological cultures should be collected, and a de-escalation applied when antimicrobial susceptibility tests are available. Standard measures to prevent infections and the identification of carriers of MDR bacteria are essential strategies to prevent infections in cirrhosis. Antibiotic prophylaxis should be applied only to gastrointestinal bleeding, SBP recurrence prevention, and cirrhotics at high risk of a first episode of SBP.
\end{abstract}

\section{Introduction}

Bacterial infections are very common in patients with cirrhosis and currently represent the main reason for hospital admission. Moreover, despite recent improvement in the management of cirrhosis, mortality due to infections in cirrhosis remains significant ${ }^{1-3}$ Both

Correspondence: Mario Mitra, ARNAS Civico-Di CristinaBenfratelli, piazza Nicola Leotta 4, 90100 Palermo, Italy. Tel.: +39.091.6662742 - Fax: +39.091.6662755.

E-mail: mario.mitra@arnascivico.it

Key words: Liver cirrhosis; bacterial infection; multiresistant bacteria.

Contributions: MM searched and reviewed literature, drafted and reviewed the paper; AM1 searched and reviewed literature, reviewed the paper; FP searched and reviewed literature, reviewed the paper; AM2 reviewed the paper.

Conflict of interests: the authors declare no potential conflict of interests.

Ethics approval and consent to participate: not required.

Received for publication: 22 April 2020.

Revision received: 31 May 2020.

Accepted for publication: 8 June 2020

This work is licensed under a Creative Commons Attribution NonCommercial 4.0 License (CC BY-NC 4.0).

${ }^{\circ}$ Copyright: the Author(s), 2020

Licensee PAGEPress, Italy

Italian Journal of Medicine 2020; 14:126-135

doi:10.4081/itjm.2020.1306 preventive measures, early recognition, and proper management are necessary to minimize morbidity and mortality of infections in cirrhosis. This paper aims to review the epidemiology, predisposing factors, and management of bacterial infections in cirrhosis.

\section{Epidemiology}

Bacterial infections prevalence is about $20-40 \%$ in hospitalized cirrhotic patients, 4-5 fold higher than hospitalized patients for other diseases..$^{2-6}$ In addition, patients with cirrhosis seem to have a 2.6 -fold higher risk of sepsis than those without it. ${ }^{7}$ The risk of bacterial infection is higher in patients with Child-Pugh $\mathrm{C}$ than both Child-Pugh A/B or with model end-stage liver disease $(M E L D)<15$. Other risk factors are the history of previous infections and gastrointestinal bleeding. ${ }^{8}$ Alcoholic etiology and alcohol consumption are risk factors for bacterial infections mainly in cirrhotic patients without advanced liver dysfunction. ${ }^{9}$

In a recent multicenter study on 1302 hospitalized patients with cirrhosis and infections, the most common infections were spontaneous bacterial peritonitis (SBP), followed by respectively urinary tract infections (UTI), pneumonia, spontaneous bacteremia, skin and soft tissue infections (SSTI). ${ }^{10}$ Similar results are reported in previous studies. ${ }^{8}$ The risk of contracting an infection during hospitalization is about $15 \%$, according to a recent series in whom infections mainly involved respiratory and urinary tract. ${ }^{11}$

Enterobacteriaceae and non-enterococcal streptococci are the leading cause of spontaneous infections. ${ }^{12}$ 
However, non-classical pathogens or multidrug-resistant (MDR) bacteria represent a new issue.

In fact, in a large prospective single-center study of cirrhotic patients with infections, MDR bacteria were isolated in respectively $4 \%, 14 \%$, and $35 \%$ of community-acquired, healthcare-associated, and nosocomial infections. Primary resistant organisms found where extended-spectrum $\beta$-lactamase (ESBL)-producing Enterobacteriaceae, followed by Pseudomonas aeruginosa, methicillin-resistant Staphylococcus aureus (MRSA) and Enterococcus faecium. Incidence of septic shock and death from infections caused by resistant bacteria were high. ${ }^{13}$

In a recent intercontinental study of 1302 patients with cirrhosis, MDR organisms were isolated in 34\% of patients with a positive culture. The most commonly isolated MDR bacteria were ESBL Enterobacteriaceae, MRSA, vancomycin-resistant enterococci, P. aeruginosa, and Acinetobacter baumannii. Extensively drug-resistant (XDR) bacteria were isolated in $8 \%$ of those with a positive culture. The most common XDR bacteria were carbapenemase-producing Enterobacteriaceae, $P$. aeruginosa, and A. baumannii. ${ }^{10}$ MDR and XDR bacterial infections are widespread in India $(73 \%$ and $33 \%$ of isolates, respectively). Whereas the prevalence of MDR infections was lower in North America (27\% and 4\% in the United States, respectively), and there was significant variability across Europe (MDR prevalence from 57\% in Israel to $17 \%$ in Russia). Rate of infections caused by MDR bacteria was correlated with young age, male sex, advanced liver failure, prolonged and recurrent use of systemic antibiotics in the previous 3 months, recent invasive procedures, and exposure to health care. Unlike previous studies, antibiotic prophylaxis for SBP with quinolones did not correlate with MDR bacterial infections. MDR bacterial infections were associated with low efficacy of empirical antibiotic treatment, a more frequent need to escalate antibiotic treatment, a prolonged antibiotic treatment, and a low rate of infection resolution. Moreover, patients with MDR bacterial infections had a higher incidence of septic shock and mortality than non-MDR. ${ }^{10}$

Another European multicenter study compared bacterial infections of a historical series of decompensated cirrhosis in 2011 with more recent series (from 2017 to 2018) to assess potential epidemiological changes. Overall, the prevalence of MDR bacterial increased from $29 \%$ in the former to $38 \%$ in the latter. ${ }^{14}$

\section{Mechanism of increased susceptibility of infections and clinical consequences}

Several factors are involved in the pathogenesis of bacterial infections in cirrhosis (Figure 1). Regarding SBP and spontaneous bacteremia, bacterial translocation from the intestinal lumen to the mesenteric lymph

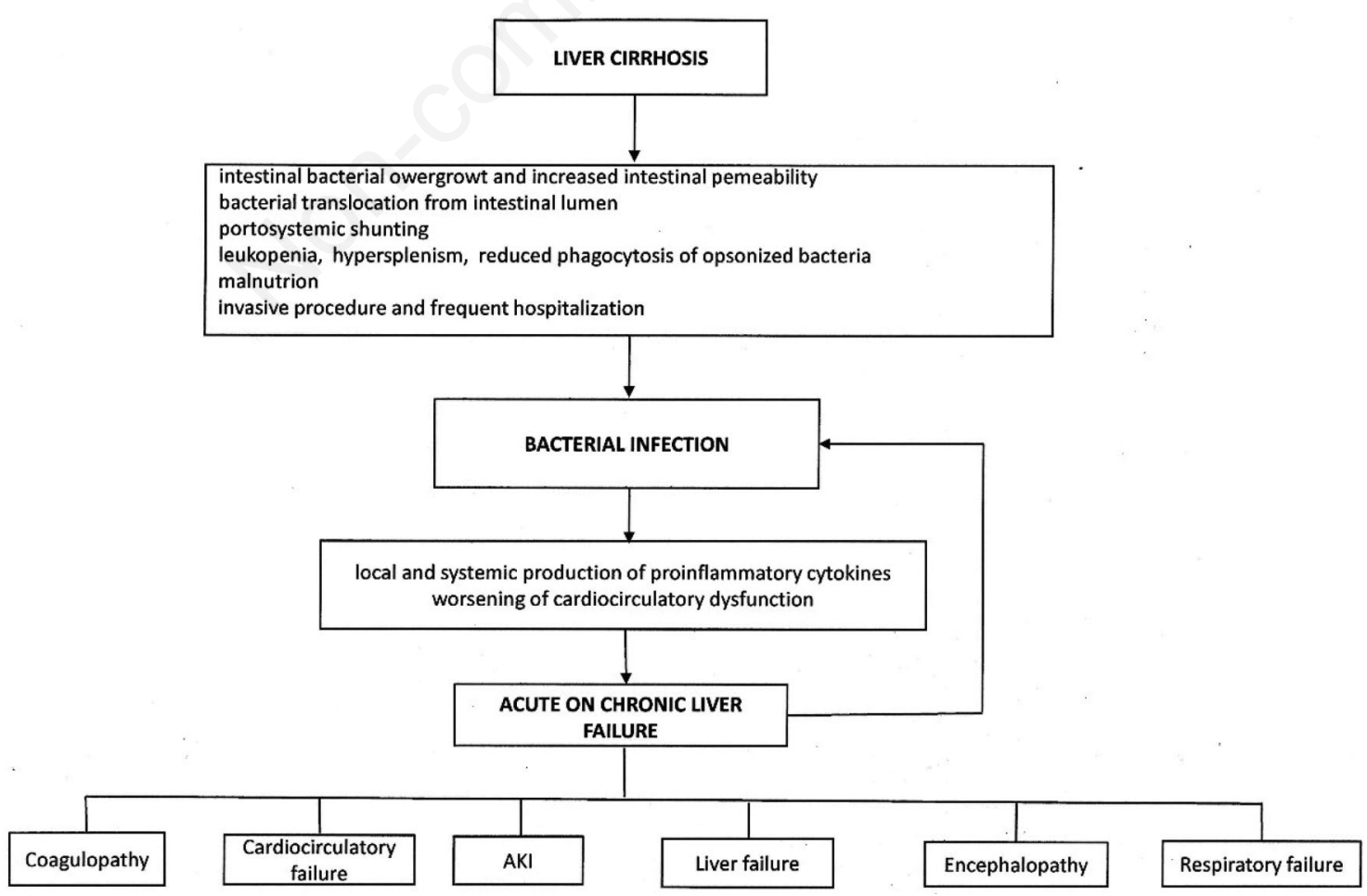

Figure 1. Pathogenetic mechanisms involved in bacterial infections. AKI, acute kidney injury. 
node is the core event. Many factors, including the quantity and quality of microbiota, increased intestinal permeability, and local defects in host immunity, can contribute to bacterial translocation. Furthermore, several abnormalities in both innate and adaptive components of the immune system response to the microbial challenge are possible in cirrhosis, leading to a state of acquired immunodeficiency. ${ }^{12,15}$

Moreover, bacterial infections promote an excessive systemic inflammatory response, resulting in tissue damage and organ hypoperfusion, so determining adverse effects on both liver disease and survival. ${ }^{12,16-}$

${ }^{18}$ In a systematic review of studies on cirrhosis with infections, the overall mortality of infected patients was significantly higher than non-infected. ${ }^{4}$

Most patients with infection can develop a multiple organ failure (liver, kidney, brain, coagulation, circulation, and lung), characterizing the so-called acute-on-chronic liver failure (ACLF). A multicenter European study on 642 patients (407 with ACLF and 235 with only acute decompensation) found that the prevalence of infections at diagnosis was significantly higher in patients with ACLF. Furthermore, nosocomial infections, infections by MDR bacteria, the severity of ACLF, and mortality were also significantly higher in patients with ACLF precipitated by bacterial infections. ${ }^{18}$ The poor prognosis of ACLF with infections has been confirmed in other 2 studies. ${ }^{19,20}$ Moreover, patients with ACLF not triggered by infections presented a higher incidence of bacterial infections during follow-up than patients with acute decompensation but without ACLF. ${ }^{18}$ Consequently, infections are a trigger of ACLF, but ACLF favors infections too because of both oxidative stress and a condition of immune paralysis, increased bacterial translocation, change of intestinal microbiota, and finally invasive therapeutic procedures.

\section{Diagnosis of infections in cirrhosis}

Signs and symptoms of infections may be very subtle in patients with cirrhosis. Early diagnosis and prompt initiation of adequate antibiotic therapy are essential in the management of patients with cirrhosis and bacterial infections, as delay and inappropriate therapy are associated with increased mortality. ${ }^{21}$ Here we discuss some suggestions that may be useful in clinical practice.

An infection should be suspected in the presence of the classic general and local symptoms or one of the following: i) new onset of portosystemic encephalopathy without obvious causes; ii) worsening of renal function; iii) white blood cell increase; iv) worsening of liver function tests. ${ }^{8}$ If an infection is suspected, patients should undergo a complete workup consisting of assessment of organ function, blood and urine culture, chest X-ray, paracentesis with fluid neutrophil count and culture if ascites is present, sputum culture if positive chest X-ray, abdominal ultrasonography, stool culture and clostridium toxin assay in case of gastrointestinal symptoms. Patients assuming steroids or immunosuppressive drugs are at risk of fungal infection and chest high-resolution computed tomography $(\mathrm{CT})$, galactomannan, and $\beta$-glucan antigen in sputum or bronchoalveolar should be assayed. Wound culture and cerebrospinal fluid culture are indicated if specific clinical signs are present.

Systemic inflammatory response syndrome (SIRS) criteria are neither specific nor sensitive for the diagnosis of infections because patients with cirrhosis may have leukopenia due to hypersplenism, tachypnea due hepatic encephalopathy or ascites, and bradycardia due to use of $\beta$-blockers. ${ }^{12}$ Sepsi-3 criteria and quick-sequential organ failure assessment score are more accurate than SIRS criteria in predicting short-term mortality in patients with cirrhosis and bacterial infections. ${ }^{22}$

Far from being a gold standard, both procalcitonin (PCT) and C-reactive protein (CRP), are useful serum markers to discriminate cirrhotic patients with or without bacterial infections. In a meta-analysis including 10 diagnostic studies (1144 cirrhotic patients and 435 bacterial infections episodes), the pooled sensitivity estimates were 79\% [95\% confidence interval (CI): 64\%-89\%] for PCT and 77\% (95\% CI: 69\%-84\%) for CRP tests, whereas the pooled specificity were $89 \%$ (95\% CI: 82\%-94\%) for PCT and 85\% (95\% CI: 76\%90\%) for CRP. The authors concluded that PCT and CRP have nearly comparable diagnostic accuracy in diagnosing bacterial infection in cirrhotic patients. ${ }^{23}$

Other serum markers, like interleukin-6, Presepsin, the fluorescence intensity of mature neutrophils, showing some correlation with infections in cirrhosis, are not currently used in clinical practice. ${ }^{24-26}$ Molecular biology tests to detect bacterial infections are promising, but there are few studies in the specific setting of cirrhosis. ${ }^{27}$

\section{Management of specific infections}

\section{Spontaneous bacterial peritonitis}

Spontaneous bacterial peritonitis is defined as a bacterial infection of ascitic fluid without any evident intra-abdominal source of infection. One-year incidence is about $3.5 \%$ in outpatients with decompensated cirrhosis, while prevalence is about $10 \%$ in hospitalized patients. ${ }^{28,29}$ Historically, mortality exceeded $90 \%$, but recently reduced to approximately $25 \%$ due to early diagnosis and treatment. ${ }^{29,30}$

Patients with SBP may have fever, abdominal pain, ileus, worsening of pre-existing ascites, encephalopathy, although symptoms may be absent in up to one- 
third of cases. Diagnostic paracentesis should be performed in all cirrhotic patients with ascites at the time of admission and/or in case of gastrointestinal bleeding, shock, signs of inflammation, hepatic encephalopathy, worsening of liver or renal function. In fact, diagnostic delay of SBP can increase hospital mortality by over $3 \%$ per hour ${ }^{31}$ and in a large, recent, retrospective study, early paracentesis within $24 \mathrm{~h}$ was associated with a reduced inpatient all-cause mortality [odds ratio (OR) $0.68,95 \%$ CI $0.63-0.73, \mathrm{P}<0.001$ ], SBP-related mortality (OR 0.84, 95\% CI 0.73-0.94, $\mathrm{P}=0.01$ ), and 30 -day readmission (OR $0.87,95 \% \mathrm{CI}$ 0.82-0.92, $\mathrm{P}<0.001){ }^{32}$

Polymorphonuclear (PMN) cells in ascitic fluid of $>250 / \mathrm{mm}$ is considered diagnostic of SBP. ${ }^{3}$ Moreover, ascites culture was reported to be negative in as many as $60 \%$ of patients with clinical manifestation suggestive of SBP and increased PMN counts ${ }^{26}$ (culture-negative SBP). These patients should be treated in a similar manner as the culture-positive SBP. Some patients have bacterascites in which cultures are positive, but PMN ascitic counts $<250 / \mathrm{mm}^{3}$. European Association for the Study of the Liver (EASL) guidelines recommended treatment in the presence of signs of infection. Otherwise, a second paracentesis should be performed. In case of culture resulting positive again, regardless of the PMN count, treatment is suggested. ${ }^{29}$ In a recent study, $42 \%$ with bacterascites was left untreated, reporting culture persisting positive or evolving to an overt SBP. ${ }^{33}$

Empirical antibiotic therapy must be initiated immediately after the diagnosis of SBP. It is important to consider separately community-acquired (CA) SBP from nosocomial (HA) SBP (diagnosis after $48 \mathrm{~h}$ of hospital admission) and healthcare-associated (any prior 90-day health care contact) SBP. Multiple studies have shown a high prevalence of MDR bacteria in nosocomial SBP. In a systematic review including 9 studies, the percentage of MDR organisms isolated in
HA-SBP varied from 36.8 to $50 \%$ for Gram-positive and from $30 \%$ to $66.6 \%$ for Gram-negative bacteria. ${ }^{34}$ A randomized controlled trial (RCT) suggested that meropenem + daptomycin is more effective than ceftazidime in the empirical treatment of nosocomial SBP (86.7\% responders vs 25\%). ${ }^{35}$

According to these data, EASL guidelines ${ }^{29}$ recommended third-generation cephalosporin as first-line antibiotic treatment for CA-SBP in countries with low rates of bacterial resistance. Meanwhile, piperacillin/ tazobactam or carbapenem should be considered in countries with high rates of bacterial resistance. Healthcare-associated or HA-SBP should be treated with piperacillin/tazobactam in areas with low prevalence di ESBL producing Enterobacteriaceae, while carbapenem should be used in areas with a high prevalence of ESBL producing Enterobacteriaceae. Carbapenem should be combined with glycopeptide or daptomycin or linezolid in areas with a high prevalence of Gram-positive MDR bacteria (Table $1^{12,29}$ ).

De-escalation, according to bacterial susceptibility based on positive cultures, is recommended to minimize resistance selection pressure. The efficacy of antibiotic therapy should be checked with a second paracentesis after $48 \mathrm{~h}$ from treatment start. The response to antimicrobials is defined as $\mathrm{a} \geq 25 \%$ reduction of PMN count. Treatment should last at least 5-7 days.

Secondary bacterial peritonitis should be suspected in case of multiple organisms on ascitic culture, very high ascitic PMN count and/or high ascitic protein concentration, or in those with an inadequate response to therapy. In patients with suspected secondary bacterial peritonitis, urgent CT scanning is mandatory.

Despite proper and early antibiotic treatment, SBP is associated with a high risk of acute kidney injury. Intravenous albumin, together with antibiotic therapy, reduces the risk of renal impairment and mortality, although this benefit was mainly observed in patients with basal serum bilirubin $\geq 4 \mathrm{mg} / \mathrm{dL}$ or serum creati-

Table 1. Empirical antibiotic treatment for bacterial infections in cirrhosis. ${ }^{12,29}$

\begin{tabular}{lll}
\hline Type of infection & Community-acquired infections & Nosocomial infections \\
\hline $\begin{array}{l}\text { SBP, SBE, } \\
\text { spontaneous } \\
\text { bacteremia }\end{array}$ & $\begin{array}{l}3^{\text {rd }} \text { generation cephalosporins } \\
\text { In countries with high rates of MDROs } \\
\text { be considered }\end{array}$ & $\begin{array}{l}\text { Piperacillin-tazobactam in areas with a low prevalence of MDROs; } \\
\text { carbapenem in areas with a high prevalence of ESBL producing } \\
\text { Enterobacteriaceae. To add glycopeptides or daptomycin or linezolid in } \\
\text { areas with a high prevalence of MDR Gram-positive }\end{array}$ \\
\hline UTI & $\begin{array}{l}\text { Uncomplicated, outpatient: ciprofloxacin or } \\
\text { cotrimoxazole }\end{array}$ & $\begin{array}{l}\text { Uncomplicated, outpatient:fosfomycin or nitrofurantoin } \\
\text { If sepsis: meropenem }+ \text { teicoplanin or vancomycin in areas with a high } \\
\text { prevalence of MDR Gram-positive }\end{array}$ \\
& piperacillin-tazobactam & \\
\hline Pneumonia & $\begin{array}{l}\text { Ceftriaxone or piperacillin-tazobactam }+ \\
\text { macrolideor levofloxacin or moxifloxacin }\end{array}$ & $\begin{array}{l}\text { Ceftazidime or meropenem }+ \text { levofloxacin } \pm \text { teicoplanin or vancomycin } \\
\text { if risk factors of MRSA }\end{array}$ \\
\hline Cellulitis & $\begin{array}{l}\text { Piperacillin-tazobactam or } 3^{\text {rd }} \text { generation } \\
\text { cephalosporins }+ \text { oxacillin }\end{array}$ & $\begin{array}{l}3^{\text {rd }} \text { generation cephalosporins or meropenem }+ \text { oxacillin or glycopeptides } \\
\text { or daptomycin or linezolid if risk factors of MRSA }\end{array}$ \\
\hline
\end{tabular}

SBP, spontaneous bacterial peritonitis; SBE, spontaneous bacterial empyema; MDRO, multidrug-resistant organism; ESBL, extended-spectrum $\beta$-lactamase; UTI, urinary tract infection; MRSA, methicillin-resistant Staphylococcus aureus. Healthcare-associated infections like nosocomial infections in case of a high prevalence of MDROs or sepsis. 
nine $\geq 1 \mathrm{mg} / \mathrm{dL} .{ }^{36}$ Current guidelines recommend the administration of albumin $1.5 \mathrm{~g} / \mathrm{kg}$ at diagnosis and 1 $\mathrm{g}$ on day 3 in patients with SBP. ${ }^{29}$

\section{Pneumonia}

Pneumonia is one of the most frequent infections in cirrhotic patients. Few studies compared pneumonia in cirrhotics and general population. In a study on community-acquired pneumonia (CAP), patients with cirrhosis presented higher pneumonia severity index, the prevalence of bacteremia, or septic shock than in the control group. Mortality was higher in cirrhotics and increased with the severity of the liver disease. Streptococcus pneumoniae and Pseudomonas aeruginosa were more frequently documented in patients with cirrhosis than in noncirrhotics, but there were no differences regarding other pathogens. ${ }^{37}$

Any cirrhotic patient presenting with fever and/or respiratory symptoms should undergo a diagnostic workup with chest ray or CT, blood cultures, sputum Gram stain and cultures, Legionella, and Streptococcus pneumonia urinary antigen tests.

EASL guidelines recommend treating CAP by a $\beta$ lactam + macrolide or monotherapy with a respiratory fluoroquinolone. The duration of treatment should be 5-7 days. For healthcare and nosocomial pneumonia, one must consider the local prevalence of MDR bacteria and factors increasing the likelihood of MRSA or Pseudomonas aeruginosa etiology. Empirical therapy includes piperacillin/tazobactam or ceftazidime or carbapenem + fluoroquinolone. A glycopeptide should be added in patients with risk factors for MRSA (Table $1^{12,29}$ ). Broad-spectrum empiric antibiotic therapy should be changed to a narrower regimen based upon respiratory and blood culture results. The use of aminoglycosides is discouraged. PCT levels, in addition to clinical criteria, are useful to guide antibiotic discontinuation. A 7-day course of therapy is often sufficient. ${ }^{12,38,39}$

\section{Urinary tract infections}

Urinary tract infections account for approximately one-third of bacterial infections in cirrhosis ${ }^{8,10}$. Usually, they are classified as upper UTI (pyelonephritis) and lower UTI (cystitis, prostatitis) depending on the site of infection. Moreover, as uncomplicated or complicated according to underlying conditions - comorbidities and functional or anatomical abnormalities of the urinary tract - increasing risk of treatment failure. The definition of complicated UTI is controversial. In the European Urology Association (EUA) guidelines, healthcare UTIs, nosocomial UTIs, and those occurring in any immunosuppressive conditions are defined as complicated. However, liver cirrhosis was not included as an immunosuppressive condition. ${ }^{40}$
Enterobacteriaceae, especially Escherichia coli, are the most common causative pathogen of UTI, ${ }^{41}$ whereas hospital-acquired UTIs are frequently due to Enterobacter, followed by Klebsiella, E. coli, Acinetobacter, $P$. aeruginosa, MRSA, with an overall MDR bacteria rate of $53.8 \% .{ }^{42}$ Notably, in a retrospective study on 108 hospitalized cirrhotics with UTI, 38\% of the patients presented a concomitant infection in other sites: SBP in $49 \%$, pneumonia in $31 \%$, bacteremia in $9 \%$, cholangitis in $4 \%$. However, UTI mortality was inferior to other sites infections. ${ }^{41}$

Empiric therapy choice should consider MDR bacteria local prevalence and be changed or de-escalated according to the bacterial culture results. Outpatients with uncomplicated CA-UTI should be treated with ciprofloxacin or cotrimoxazole, whilst fosfomycin or nitrofurantoin for HA-UTI. Hospitalized patients or in case of sepsis, CA-UTI should be treated with $3^{\text {rd }}$ generation cephalosporin or piperacillin-tazobactam. In severe HA-UTI, considering the high prevalence of MDR bacteria, an association of carbapenem with glycopeptide is indicated ${ }^{29,43,44}$ (Table $1^{12,29}$ ). According to a meta-analysis of RCTs in general patients, seven days seem equivalent to longer treatment. ${ }^{45}$

Asymptomatic bacteriuria without clinical evidence of infection should not prompt antibiotic treatment in order to reduce the development of antibiotic resistance. Exceptions to this rule are pregnant women, diabetic patients and cirrhotic patients undergoing urinary tract maneuvers. ${ }^{46}$

\section{Skin and soft tissue infections}

Skin and soft tissue infections are about $8 \%$ of all bacterial infections in cirrhosis. ${ }^{10}$ Furthermore, a nationwide population-based study in Taiwan showed that cirrhotic patients are at increased risk of cellulitis, ${ }^{47}$ due to: fragile, thin and edematous skin, poor hygiene, malnutrition, frequent hospitalization, and invasive procedures. Usually, the most common SSTI in cirrhotic patients affects the lower extremities.

SSTI diagnosis is mainly clinical, and a bacterial diagnosis from blood or skin aspirates has been reported at a variable rate between $30-80 \% .^{45,47,48}$ As opposed to cellulitis in the general population, where the dominant etiological organisms are Gram-positive bacteria (Streptococci and S. aureus) ${ }^{46}$ Gram-negative organisms (E. coli, Klebsiella, Pseudomonas) are reported in 70-80 \% in cirrhosis. ${ }^{45,46}$ However a recent study indicated Gram-negative bacteria prevalence only in decompensated but not compensated cirrhosis. ${ }^{49}$

Advanced liver disease, MELD score $>15$, serum albumin $<2.5 \mathrm{~g} / \mathrm{dL}$ are the major liver-related risk factors for cellulitis. ${ }^{49-51}$

No RCTs for antibiotic therapy of cellulitis in a cirrhotic setting where published. Anyway, as observation- 
ally reported, initial empirical therapy with cephalosporin or amoxicillin-clavulanic acid or piperacillin-tazobactam should be suggested, but a switch therapy for failure or microbiological guide is necessary in $1 / 3$ of cases. ${ }^{49-51}$ Furthermore, EASL guidelines recommend empirical antimicrobial therapy in community-acquired skin and soft tissue infection by piperacillin-tazobactam or $3^{\text {rd }}$ generation cephalosporin + oxacillin, while in the setting of healthcare or nosocomial infection, $3^{\text {rd }}$ generation cephalosporin or meropenem + oxacillin or glycopeptide or daptomycin or linezolid ${ }^{27}$ (Table $1^{12,29}$ ). Treatment should last an average of 7 days. Depending on the clinical response, therapy can be further expanded. In purulent forms, incision and drainage are indicated. ${ }^{48}$

\section{Bloodstream infection}

Bloodstream infections (BSI) account for 5-8\% of all infections in liver cirrhosis. ${ }^{10,14}$ In a populationbased study, BSI was reported as 10 times more common in cirrhotics than in non-cirrhotics. ${ }^{52}$ In a large retrospective study, BSI incidence in hospitalized cirrhosis was estimated in 11.7 per 10,000 patient-days with a 30-day mortality rate of $29 \% .{ }^{53}$ Similar mortality rates where reported in other studies..$^{54,55}$

BSI are heterogeneous infections. Most of BSI episodes are healthcare-associated or $\mathrm{HA}^{53-56}$ and the source of infection was identified in 22 to $85 \%$ of the cases. ${ }^{53-57}$ When the infection site is identified, the intra-abdominal source is the most common. ${ }^{46,55}$ Indicators of poor prognosis are intra-abdominal source, concomitant septic shock, administration of inappropriate antibiotics within $24 \mathrm{~h}$, and liver failure. ${ }^{53-55}$ Chronic liver failure-organ failure and MELD scores predict 28-day mortality better than SIRS score. ${ }^{57}$

Gram-negative bacteria are responsible for $50-70 \%$ of BSIs, and the most frequently observed are E. coli and $K$. pneumonia. Staphylococci and streptococci are the most prevalent Gram-positive pathogens. ${ }^{53-56,58}$ The prevalence of MDR bacteria is about $30 \%$, and BSIs by MDR pathogens are associated with poor prognosis. ${ }^{53,55,56}$ Candida was reported in $5-10 \%$ of BSI, and correlated with the worst mortality rates. ${ }^{53,58}$

The initial antimicrobial choice should take into account local epidemiology, site of infection onset, and an individual patient risk factor for antimicrobial-resistant infection (e.g., prior antibiotic exposure, colonization status). In the setting of healthcare or HA-BSI $3^{\text {rd }}$ generation cephalosporins and fluoroquinolones should be avoided. If the local prevalence of ESBL producing strains is low, piperacillin/ tazobactam can be used. Where it is high, meropenem is suggested. In areas with a high prevalence of MRSA or in patients with suspected devicerelated infection, it is recommended to add glycopeptide (Table $1^{12,29}$ ).

\section{Spontaneous bacterial empyema}

About $2-2.4 \%$ of patients with cirrhosis present hydrothorax. Infection of pre-existing hepatic hydrothorax, known as spontaneous bacterial empyema (SBE), occurs in $13 \%$ to $16 \%$ of patients, and it is associated with a mortality of $38 \%$. SBE is associated with SBP in about $50 \%$ of cases.

The diagnosis is based on pleural fluid analysis obtained by diagnostic thoracentesis. The diagnosis of SBE is established when the pleural fluid analysis shows a positive culture and PMN $>250 / \mathrm{mm}^{3}$ and likely when negative culture and PMN $>500 / \mathrm{mm}^{3}$, in the absence of pneumonia. Pleural fluid culture is positive in about $75 \%$ of cases.

Management of antimicrobial therapy is the same as the SPB and the only indication for chest-tube drainage is the presence of pus in the pleural space. ${ }^{29,59}$

\section{Proton pump inhibitors and the risk of infections in cirrhosis}

Cirrhotic patients have a significantly higher risk of peptic ulcer bleeding. ${ }^{60}$ Most patients take proton pump inhibitors (PPIs) chronically, sometimes with an overuse. A state of gastric acid suppression induced by PPIs is known to be associated with small bowel bacterial overgrowth, alteration of gut flora, reduction of gastrointestinal motility and impairment of neutrophil function. ${ }^{61-63}$

Several studies suggested that PPIs are associated with increased risk of bacterial infections in cirrhotic patients. In a meta-analysis of 10 case-control studies and 6 cohort studies, PPI use was associated with SBP only in case-control studies, but not in the cohort studies. PPI therapy was not associated with mortality ${ }^{64}$ Other most recent studies have highlighted the negative effect of PPIs in cirrhotic patients, although with heterogeneous results. ${ }^{65-76}$ Numerous confounding factors can contribute to these results: cirrhotics with PPI therapy often have a previous gastrointestinal bleeding or advanced liver disease, two conditions associated with increased risk of infection and mortality, or assume antiplatelet drug for concomitant cardiovascular disease. PPI therapy has also been linked to increased risk of Clostridium difficile infection and hepatic encephalopathy. ${ }^{71-74}$ Another unresolved question is whether it is advisable to reduce PPI dose in cirrhotics because all PPIs are metabolized by liver. ${ }^{75}$

Based on the results of the available studies, prolonged use of PPI should be adopted with caution and PPI should be used only if clinically indicated.

\section{Prophylaxis of infections in patients with cirrhosis}

Because bacterial translocation from the gut is one of the main pathogenetic mechanisms in the develop- 
ment of bacterial infection in cirrhosis, the use of an antimicrobial agent to prevent infections represents a suggestive strategy.

Currently, antimicrobial prophylaxis is recommended under three conditions: i) after an episode of SBP; ii) in patients with variceal bleeding; and iii) in patients at high risk of developing SBP.

Recurrence after the first episode of SBP is very high $\left(69 \%\right.$ at 1 -year) ${ }^{77}$ In a randomized controlled trial, the administration of norfloxacin $400 \mathrm{mg}$ per day resulted more effective than placebo in preventing the recurrence of SBP from $68 \%$ to $20 \%$ after 1 -year. ${ }^{78}$ In a recent retrospective cohort study, SBP recurrence rate in the patient receiving norfloxacin remained similar. $^{79}$ Therefore, secondary prophylaxis of SBP with norfloxacin should be given indefinitely or until liver transplant.

Patients with variceal bleeding have a high risk of developing bacterial infections. Approximately 25$65 \%$ of patients, in the absence of antimicrobial prophylaxis, present or develop infections within the first seven days of bleeding, which in turn increased the risk of rebleeding and hospital mortality. ${ }^{8}$ A metaanalysis, including twelve trials, reported that antibiotic prophylaxis for upper gastrointestinal bleeding reduced bacterial infections and mortality. ${ }^{80}$ Then, all cirrhotic patients with gastrointestinal bleeding should receive antibiotic therapy. In patients with advanced liver disease, those already on quinolone prophylaxis, and in a hospital setting with a high prevalence of quinolone-resistant infection, $3^{\text {rd }}$ generation cephalosporins are preferable than oral quinolones to prevent infections during gastrointestinal bleeding. The antibiotic therapy should be started as soon as possible with a 7-day course.

In cirrhotic patients with a high risk of SBP (ascitic fluid protein concentration $<1.5 \mathrm{~g} / \mathrm{dL}$, Child-Pugh score $\geq 9$ and at least one of the following criteria: serum bilirubin $\geq 3 \mathrm{mg} / \mathrm{dL}$, serum creatinine level $\geq 1.2 \mathrm{mg} / \mathrm{dL}$ or serum sodium $\leq 130 \mathrm{mEq} / \mathrm{L}$ ), daily long term administration of norfloxacin $400 \mathrm{mg}$ was more effective than placebo in preventing the first episode of SBP and reduced mortality. Weekly ciprofloxacin $(750 \mathrm{mg})$ administration is reported as an alternative option, but survival benefit did not reach the significance threshold. ${ }^{81}$

The most pressing concern associated with antibiotic prophylaxis is the development of antibiotic resistance. In some observational studies, long term use of norfloxacin was associated with a higher rate of MDR bacterial infections. ${ }^{6,13,82-84}$ This effect has not been confirmed in a recent worldwide epidemiological study. ${ }^{10}$ Rifaximin, a non-absorbable antibiotic, also appears effective for primary and secondary prophylaxis of SBP in some low-quality studies. ${ }^{80-83}$ Moreover, long-term use of rifaximin did not associate with a higher prevalence of MDR bacterial infections in a study. ${ }^{85}$ If these results will be confirmed, the use of rifaximin for SBP prophylaxis could simplify medication regimens overall in patients with hepatic encephalopathy.

Based on available evidence, antibiotic prophylaxis should be restricted to subgroups of cirrhotics at a very high risk of infection.

Another strategy to prevent infections could be the long-term use of albumin. In an open-label randomized trial in cirrhotics with ascites, long-term human albumin administration $40 \mathrm{~g}$ twice weekly for 2 weeks, and then $40 \mathrm{~g}$ weekly, in addition to standard medical therapy, significantly reduced the incidence of SBP and other infections, hepatorenal syndrome, hepatic encephalopathy, and mortality. ${ }^{86}$ Anyway, long-term albumin administration seems not to be feasible for all patients for both economic and logistical reasons.

\section{Conclusions}

Bacterial infections are a relevant complication of cirrhosis. Early diagnosis and treatment are crucial to improving prognosis.

Prescription of empiric antibiotics should be made according to the severity of infection, the presence or absence of risk factors for the development of MDR bacteria, and the local epidemiology. Only EASL issued formal recommendations other than SBP.

Several tools to optimize antimicrobial therapy will need to be investigated, including the role of carbapenem sparing drugs (e.g., cephalosporin- $\beta$-lactamase inhibitor combinations) in cirrhosis setting and biomolecular methods to assist clinicians in rapid detection of infections and guide antimicrobial therapy.

\section{References}

1. Schmidt ML, Barritt AS, Orman ES, et al. Decreasing mortality among patients hospitalized with cirrhosis in the United States from 2002 through 2010. Gastroenterology 2015;148:967-77.

2. Dionigi E, Garcovich M, Borzio M, et al. Bacterial infections change natural history of cirrhosis irrespective of liver disease severity. Am J Gastroenterol 2017;112: 588-96.

3. Fricker ZP, Mukthinuthalapati VVPK, Akinyeye S, et al. MELD-Na is more strongly associated with risk of infection and outcome than other characteristics of patients with cirrhosis. Dig Dis Sci 2020 [Epub ahead of print].

4. Arvaniti V, D'Amico G, Fede G, et al. Infections in patients with cirrhosis increase mortality four-fold and should be used in determining prognosis. Gastroenterology 2010;139:1246-56.

5. Rosa H, Silverio AO, Perini RF, et al. Bacterial infection in cirrhotic patients and its relationship with alcol. Am J Gastroenterol 2000;95:1290-3. 
6. Fernandez J, Navasa M, Gomez J, et al. Bacterial infections in cirrhosis: epidemiological changes with invasive procedures and norfloxacina prophylaxis. Hepatology 2002;35:140-8.

7. Foreman MG, Mannino DM, Moss M. Cirrhosis as a risk factor for sepsis and death: analysis of the National Hospital Discharge Survey. Chest 2003;124:1016-20.

8. Fagiuoli S, Colli A, Bruno R, et al. Management of infections in cirrhotic patients: report of a consensus conference. Dig Liv Dis 2014;46:204-12.

9. Gustot T, Fernandez J, Szabo G, et al. Sepsis in alcoholrelated liver disease. J Hepatol 2017;67:1031-50.

10. Piano S, Singh V, Caraceni P. Epidemiology and effects of bacterial infections in patients with cirrhosis worldwide. Gastroenterology 2019;156:1368-80.

11. Bajaj JS, O'Leary JG, Tandon P, et al. Nosocomial infection are frequent and negatively impact outcome in hospitalized patients with cirrhosis. Am J Gastroenterol 2019;114:1091-100.

12. Jalan R, Fernandez J, Wiest R, et al. Bacterial infections in cirrhosis: a position statement based on the EASL special conference 2013. J Hepatol 2014;60:1310-24.

13. Fernandez J, Acevedo J, Castro M, et al. Prevalence and risk factor of infections by multiresistant bacteria in cirrhosis: a prospective study. Hepatology 2012;55:1551-61.

14. Fernandez J, Prado V, Trebicka J, et al. Multidrug-resistant bacterial infections in patients with decompensated cirrhosis and with acute-on-chronic liver failure in Europe. J Hepatol 2019;70:398-411.

15. Piano S, Rocca A, Mareso S, Angeli P. Infections complicating cirrhosis. Liv Int 2018;38:126-33.

16. Moreau R, Jalan R, Gines P, et al. Acute-on-chronic failure is a distinct syndrome that develope in patients with acute decompensation of cirrhosis. Gastroenterology 2013;144:1426-37.

17. Piano S, Tonon M, Vettore E, et al. Incidence, predictors and outcome of acute-on-chronic liver failure. J Hepatol 2017;67:1177-84.

18. Fernandez J, Acevedo J, Wiest R, et al. Bacterial and fungal infections in acute-on-chronic liver failure: prevalence, characteristics and impact on prognosis. Gut 2018;67:1870-80.

19. Mucke MM, Ruyantseva T, Mucke VT, et al. Bacterial infection-triggered acute-on-chronic liver failure is associated with increased mortality. Liver Int 2018;38: 645-53.

20. Cao ZJ, Liu YH, Zhu CW, et al. Bacterial infection triggers and complicates acute-on-chronic liver failure in patients with hepatitis B virus-decompensated cirrhosis: a retrospective cohort study. World J Gastroenetrol 2020;26:645-56.

21. Arabi YM, Dara SI Memish Z, et al. Antimicrobial therapeutic determinants of outcomes from septic shock among patients with cirrhosis. Hepatology 2012;56: 2305-15.

22. Piano S, Bartoletti M, Tonon M, et al. Assessment of Sepsis-3 criteria and quick SOFA in patients with cirrhosis and bacterial infections. Gut 2018;67:1892-9.

23. Lin KH, Wang FL, Wu MS, et al. Serum procalcitonin and C-reactive protein levels as markers of bacterial infection in patients with liver cirrhosis: a systematic review and meta-analysis. Diagn Microbiol Infect Dis 2014;80:72-8.
24. Papp M, Torani T, Vitalis Z, et al. Presepsin teardown pitfalls of biomarkers in the diagnosis and prognosis of bacterial infection in cirrhosis. World J Gatroeneterol 2016;22:9172-85.

25. Wang H, Li Y, Zhang F, et al. Combination of PCT, sNFI and $\mathrm{dCHC}$ for the diagnosis of ascites infection in cirrhotic patients. BMC Infect Dis 2018;18:389.

26. Lin S, Huang Z, Wang M, et al. Interleukin-6 as an early markers for bacterial sepsis in patients with liver cirrhosis. J Crit Care 2015;30:732-8.

27. Sinha M, Jupa J, Mach H, et al. Emerging technologies for molecular diagnosis of sepsis. Clin Microbiol Rev 2018;31:e00089-17.

28. Rimola A, Garcia-Tsao G, Navasa M, et al. Diagnosis, treatment, and prophylaxis of bacterial peritonitis: a consensus document. International Ascites Club. J Hepatol 2000:32:142-53.

29. European Association for The Study of the Liver. EASL clinical practice guidelines for the management of patients with decompensated cirrhosis. J Hepatol 2018;69:406-60.

30. Prat LI, Wilson P, Freeman SC, et al. Antibiotic treatment for spontaneous bacterial peritonitis in people with decompensated liver cirrhosis: a network meta-analysis. Cochrane Datab Sist Rev 2019;9:CD013120.

31. Kim JJ, Tsukamoto MM, Mathur K, et al. Delayed paracentesis is associated with increased in-hospital mortality in patient with spontaneous bacterial peritonitis. Am J Gastroenterol 2014;109:1436-42.

32. Rosenblatt R, Tafesh Z, Shen N, et al. Early paracentesis in high-risk hospitalized patients: time for a new quality indicator. Am J Gastroenterol 2019;114:1863-9.

33. Oey RC, Van Buuren HR, De Jong DM, et al. Bacterascites: a study of clinical features, microbiological findings and clinical significance. Liv Int 2018;38: 2199-209.

34. Fiore M, Sansone P, Passavanti MB, et al. Nosocomial spontaneous bacterial peritonitis antibiotic treatment in the era of multi-drug resistance pathogens: a systematic review. World J Gastroenterol 2017;23:4654-60.

35. Piano S, Fasolato S, Salinas F, et al. The empirical antibiotic treatment of nosocomial spontaneous bacterial peritonitis: results of a randomized, controlled clinical trial. Hepatology 2016;63:1299-309.

36. Salerno F, Navickis RS, Wilkes MM. Albumin infusion improves outcomes of patients with spontaneous bacterial peritonitis: a metanalysis of randomized trials. Clin Gastroenterol Hepatol 2013;11:123-30.

37. Viasus D, Garcia-Vidal C, Castellote J, et al. Community-acquired pneumonia in patients with liver cirrhosis. Clinical features, outcomes, and usefulness of severity scores. Medicine 2011;90:110-8.

38. Metlay JP, Waterer GW, Long AC, et al. Diagnosis and treatment of adult with community-acquired pneumonia. Am J Respir Crit Care Med 2019;200:e45-e67.

39. Kalil AC, Metersky ML, Klompas M, et al. Management of adults with hospital-acquired and ventilator-associated pneumonia: 2016 clinical practice guidelines by Infectious Disease Society of America and the American Thoracic Society. Clin Inf Dis 2016:63:e61-e111.

40. Johansen TE, Botto H, Cek M, et al. Critical review of current definitions of urinary tract infections and proposal of an EAU/ESIU classification system. Int J Antimicrob Agents 2011;38:s64-70. 
41. Reuken PA, Stallmach A, Bruns T. Mortality after urinary tract infections in patients with advanced cirrhosis. Relevance of acute injury and comorbitities. Liv Int 2013;33:220-30.

42. Milovanovic T, Dumic I, Veličkovic J, et al. Epidemiology and risk factor for multidrug resistant hospital-acquired urinary tract infection in patient with liver cirrhosis: a single center experience in Serbia. BMC Infect Dis 2019;19:141.

43. European Association of Urology. EAU Guidelines: Urological Infections. Amhem, Netherlands: European Association of Urology. Available from: uroweb.org/ guideline/urological-infections. Accessed: March 11, 2020.

44. Gupta K, Hooton TM, Naber KG, et al. International practice guidelines for the treatment of acute uncomplicated cystitis and pyelonephritis in women: a 2010 update by the Infectious Disease Society of America and the European Society for Microbiology and Infectious Diseases. Clin Infect Dis 2011:52:e103-e120.

45. Eliakim-Raz, Yahav D, Paul M, Leibovici L. Duration of antibiotic treatment for acute pyelonephritis and septic urinary tract infection-7 days or less versus longer treatment: systematic review and meta-analysis of randomized controlled trials. J Antimicrobiol Chemother 2013;68:2183-91.

46. Nicolle LE, Gupta K, Bradley SF, et al. Clinical practice guidelines for the management of asymptomatic bacteriuria: 2019 update by Infectious Disease Society of America. Clin Infect Dis 2019;68:1611-5.

47. Lin MN, Tsai CC, Hung TH, et al. The risk of cellulitis in cirrhotic patients: a nationwide population-based study in Taiwan. Gut Liver 2012;6:482-5.

48. Stevens DL, Bisno AL, Chambers HL, et al. Practice guidelines for the diagnosis and management of skin and soft tissue infections: 2014 update by the Infectious Society of America. Clin Infect Dis 2014;59:147-59.

49. Mohan P, Ramu B, Bhaskar E, et al. Prevalence and risk factor for bacterial skin infection and mortality in cirrhosis. Ann Hepatol 2011;10:15-20.

50. Hamza RE, Villyoth MP, Peter G, et al. Risk factor of cellulitis in cirrhosis and antibiotic prophylaxis in preventing recurrence. Ann Gastroenterol 2014;27:374-9.

51. Hassan EA, El-Din Abdel Renim AS, Abdel-Malek MO, et al. Are there differences in risk factors, microbial aspects, and prognosis of cellulitis between compensated and decompensated hepatitis $\mathrm{C}$ virus-related cirrhosis? Clin Mol Hepatol 2019:25:317-25.

52. Thulstrup AM, Sorensen HT, Schonheyder HC, et al. Population-based study of the risk and short-term prognosis for bacteremia in patients with liver cirrhosis. Clin Inf Dis 2000;31:1357-61.

53. Bartoletti M, Giannella M, Caraceni P, et al. Epidemiology and outcomes of bloodstream infection in patient with cirhhosis. J Hepatol 2014;2014; 61:51-8.

54. Park H, Jang KJ, Park SH, et al. Appropriate empirical antibiotic use and 30-d mortality in cirrhotic patients with bacteremia. World J Gastroenterol 2015;28:3587-92.

55. Xie Y, Tu B, Zhang X, et al. Bacterial distributions and prognosis of blood stream infections in patients with liver cirrhosis. Sci Rep 2017;7:11482.

56. Addo Smith JN, Yau R, Russo HP, et al. Bacteremia in patients with liver cirrhosis: prevalence and predictors of multidrug resistant organism. J Clin Gastroenterol 2018;52:648-54.

57. Zhao H, Gu X, Zhao R, et al. Evaluation of prognostic scoring systems in liver cirrhosis patients with blood stream infections. Medicine 2017;96:50.

58. Brandolini M, Corbella M, De Silvestro A, et al. Epidemiological characteristics of bloodstream infections in patients with different degrees of liver disease. Infection 2015;43:561-7.

59. Lv Y, Han G, Fan D. Hepatic hydrothorax. Ann Hepatol 2018;17:33-46.

60. Luo JC, Leu HB, Huang CC, et al. Cirrhotic patients at increased risk of peptic ulcer bleeding: a nationwide population-based cohort study. Aliment Pharmacol Ther 2012;36:542-50.

61. Lewis SJ, Franco S, Young G, O'Keefe SJ. Altered bowel function and duodenal bacterial overgrowt in patient treated with omeprazole. Aliment Pharmacol Ther 1996;10:557-61.

62. Lo WK, Chan WW. Proton pump inhibitor use and the risk of small intestinal overgrowth: a meta-analysis. Clin Gastroenetrol Hepatol 2013;11:483-90.

63. Zedtwitz-Liebenstein K, Wenisch C, Patruta S, et al. Omeprazole treatment diminishes intra- and extracellular neutrophil reactive oxygen production and bactericidal activity. Crit Care Med 2002;30:1118-22.

64. Yu T, Tang Y, Jiang L, et al. Proton pump inhibitor therapy and its association with spontaneous bacterial peritonitis incidence and mortality: a meta-analysis. Dig Liv Dis 2016;48:353-9.

65. Dultz G, Piiper A, Zeuzem S, et al. Proton pump inhibitors treatment is associated with the severity of liver disease and increased mortality in patient with cirrhosis. Aliment Pharmacol Ther 2015;41:459-66.

66. Kim JH, Lim KS, Min YW, et al. Proton pump inhibitors do not increase the risk for recurrent spontaneous bacterial peritonitis in patients with cirrhosis. J Gastroenterol Hepatol 2017;32:1064-70.

67. Lazaro-Pacheco IB, Servin-Caamano AI, Perez-Hernandez JL, et al. Proton pump inhibitors increase the overall risk of developing bacterial infections in patients with cirrhosis. Arq Gastroenterol 2018;55:28-32.

68. Hung TH, Tseng CW, Lee HF, et al. Effect of proton pump inhibitors on mortality in patients with cirrhosis and spontaneous bacterial peritonitis. Ann Hepatol 2018;17:933-9.

69. De Roza MA, Kai L, Kam JW, et al. Proton pump inhibitors increase mortality and hepatic decompensation in liver cirrhosis. World J Gastroenterol 2019;25:4933-44.

70. Tergast TL, Wranke A, Laser H, et al. Dose-depend impact of proton pump inhibitors on the clinical course of spontaneous bacterial peritonitis. Liver Int 2018;38:1602-13.

71. Dam G, Vilstrup H, Watson H, et al. Proton pump inhibitors as a risk factor for hepatic encephalopathy and spontaneous bacterial peritonitis in patients with cirrhosis with ascites. Hepatology 2016;64:1265.

72. Bajaj JS, Ananthakrishnan AN, Hafeezullah M, et al. Clostridium difficile is associated with poor outcomes in patients with cirrhosis: a national and tertiary center perspective. Am J Gastroenterol 2010;105:106-13.

73. Smith EZ, Northup PG, Argo CK. Predictors of mortality in cirrhosis inpatients with clostridium difficile infection. J Clin Gastroenterol 2018;52:747-51. 
74. Yan D, Huang YD, Chen YB, et al. Risk factors for Clostridium difficile infection in cirrhotic patients. Hepatobiliary Pancreat Dis Int 2019;18:237-41.

75. Weersink RA, Bouma M, Burger DM, et al. Safe use of proton pump inhibitors in patients with cirrhosis. $\mathrm{Br} \mathrm{J}$ Clin Pharmacol 2018;84:1806-20.

76. Janka T, Tonei T, Borbely B, et al. Deleterious effect of proton pump inhibitors on the disease course of cirrhosis. Eur J Gastroenterol Hepatol 2020;32:257-64.

77. Titò L, Rimola A, Gines P, et al. Recurrence of spontaneous bacterial peritonitis in cirrhosis: frequency and predictive factors. Hepatology 1988;8:27-31.

78. Gines P, Rimola A, Planas R, et al. Norfloxacin prevents spontaneous bacterial peritonitis recurrence in cirrhosis: results of a double-blind, placebo-controlled trial. Hepatology 1990;12:716-24.

79. Marciano S, Dirchwolf M, Diaz JM, et al. Spontaneous bacterial peritonitis recurrence in patients with cirrhosis receiving secondary prophylaxis with norfloxacin. Eur J Gastroenterol Hepatol 2019;31:540-6.

80. Chavez-Tapia NC, Barientos-Gutierrez T, Tellez-Avila F, et al. Meta-analysis: antibiotic prophylaxis for cirrhotic patients with upper gastrointestinal bleeding - an updated Cochrane review. Aliment Pharmacol Ther 2011;34:509-18
81. Facciorusso A, Pagiouvanni I, Cela M, et al. Comparative efficacy of long term antibiotic treatment in the primary prophylaxis of spontaneous bacterial peritonitis. Liver Int 2019;39:1448-58.

82. Goel A, Rahim U, Nguyen LH, et al. Systematic review with meta-analysis: rifaximin for the prophylaxis of spontaneous bacterial peritonitis. Aliment Pharmacol Ther 2017;46:1029-36.

83. Salehi S, Tranah TH, Lim S, et al. Rifaximin reduces the incidence of spontaneous bacterial peritonitis, variceal bleeding and all-cause admissions in patients on the liver transplant waiting list. Aliment Pharmacol Ther 2018;50:435-41.

84. Fernandez J, Tandon P, Mensa J, Garcia-Tsao G. Antibiotic prophylaxis in cirrhosis: good and bad. Hepatology 2016;63:2019-31.

85. Tandon P, Delisle A, Topal JE, Garcia-Tsao G. High prevalence of antibiotic-resistant bacterial infections among patients with cirrhosis at a US Liver Center. Clin Gastroenterol Hepatol 2012;10:1291-8.

86. Caraceni P, Riggio O, Alessandria C, et al. Long-term albumin administration in decompensated cirrhosis (ANSWER): an open-label randomized trial. Lancet 2018;391:2417-29. 